\title{
ESTUDIO DE CORRELACIÓN ENTRE LOS DIAGNÓSTICOS DE CINTURA HIPERTRIGLICERIDÉMICA Y SÍNDROME METABÓLICO EN ADULTOS DE TRUJILLO, PERÚ
}

\author{
Edgardo Linares-Reyes ${ }^{1, a}$, Karen Castillo-Minaya2,b, María Ríos-Mino,c, Jorge Huamán-Saavedra ${ }^{4, d}$
}

\begin{abstract}
RESUMEN
Objetivos. Determinar la concordancia entre la cintura hipertrigliceridémica (CHTG) y el síndrome metabólico (SM) en adultos de Trujillo según criterios diagnósticos. Materiales y métodos. Estudio observacional, prospectivo y de corte transversal en un muestra de 500 personas adultas de Trujillo, seleccionadas mediante muestreo probabilístico y estratificado (por sexo y edad). Se recopiló información consistente en medidas antropométricas, presión arterial y pruebas bioquímicas; luego se aplicó los criterios propuestos por el ATP III y Joint Interin Statement (JIS) para SM, así como los de Gómez y JIS para CHTG. Se usó el programa EPIDAT, donde se calculó la frecuencia, test Z, prueba de T Student y el test de Kappa (k) con un intervalo de confianza (IC) del $95 \%$. Resultados. Se recogió información concerniente a 239 varones y 261 mujeres. La frecuencia de CHTG según criterios de Gómez fue $30 \%$ en el total de la muestra, $29,7 \%$ en varones y $30,3 \%$ en mujeres. Según criterios JIS la frecuencia fue $34 \%$ en el total de población, $38,1 \%$ en varones y $30,3 \%$ en mujeres. EI SM según criterios JIS tuvo una buena concordancia con CHTG según criterios de Gómez con k=0,63 y según criterios JIS con k=0,66, en el total de población. Conclusiones. Se evidenció buena concordancia entre la CHTG según ambos criterios y SM según JIS.
\end{abstract}

Palabras clave: Cintura hipertrigliceridémica; Síndrome X metabólico; Obesidad abdominal; Hipertrigliceridemia (fuente: DeCS BIREME).

\section{STUDY OF CORRELATION BETWEEN DIAGNOSTIC HYPERTRIGLYCERIDEMIC WAIST AND METABOLIC SYNDROME IN ADULTS IN TRUJILLO, PERU}

\begin{abstract}
Objectives. To determine the correlation between hypertriglyceridemic waist (HTGW) and metabolic syndrome (MS) in adults of Trujillo according to diagnostic criteria. Materials and methods. Observational, prospective and cross-sectional study with a sample of 500 adults in Trujillo, selected by probability sampling and stratified by sex and age. Information on anthropometric measurements, blood pressure and biochemical tests was collected; Criteria proposed by the ATP III and Joint Interim Statement (JIS) for MS, as well as Gomez and JIS for HTGW were applied. EPIDAT software was used to calculate $Z$ test, Student $t$ test and the Kappa (k) with a confidence interval $(\mathrm{Cl})$ of $95 \%$. Results. Information regarding 239 men and 261 women were collected. The frequency of HTGW according to Gomez criteria was $30 \%$ in the total sample, $29.7 \%$ in men and $30.3 \%$ women. According to JIS criteria the frequency was $34 \%$ in the total sample, $38.1 \%$ in men and $30.3 \%$ women. Metabolic Syndrome with JIS criteria had good agreement with HTGW according to Gomez criteria with $\mathrm{k}=0.63$ and according to JIS criteria with $\mathrm{k}=0.66$. Conclusions. Good agreement was evidenced between the HTGW according to Gomez and JIS criteria and MS according to JIS.
\end{abstract}

Key words: Hypertriglyceridemic waist; Metabolic syndrome X; Obesity, abdominal; Hypertriglyceridemia (source: MeSH NLM).

\footnotetext{
Centro de Atención Primaria Metropolitano. ESSALUD. Trujillo. Perú.

Escuela de Posgrado, Universidad Nacional de Trujillo. Trujillo, Perú.

Centro de Salud Pampas-Pallasca. Ancash, Perú.

Facultad de Medicina, Universidad Nacional de Trujillo. Trujillo, Perú.

Médico cirujano; ${ }^{\mathrm{b}}$ médico cirujano, magíster en Epidemiología; ${ }^{\mathrm{C}}$ médico serumista; ${ }^{\mathrm{d}}$ patólogo clínico, doctor en Medicina.

Recibido: 20-12-13 Aprobado: 23-04-14
}

Citar como: Linares-Reyes E, Castillo-Minaya K, Ríos-Mino M, Huamán-Saavedra J. Estudio de correlación entre los diagnósticos de cintura hipertrigliceridémica y síndrome metabólico en adultos de Trujillo, Perú. Rev Peru Med Exp Salud Publica. 2014;31(2):254-60. 


\section{INTRODUCCIÓN}

El síndrome metabólico (SM) es un complejo de factores de riesgo relacionados con la enfermedad cardiovascular y diabetes mellitus. Estos factores de riesgo son: hiperglicemia, presión arterial elevada, hipertrigliceridemia, HDL bajo y obesidad central, descrito inicialmente por Reaven ${ }^{(1,2)}$. Las personas con SM tienen dos veces más el riesgo de desarrollar enfermedad cardiovascular dentro de 5 a 10 años, y cinco veces de padecer diabetes mellitus ${ }^{(3,4)}$. Los criterios del SM han sido establecidos por el Adult Treatment Panel III Report (ATP III) por la presencia de, al menos, tres de cinco factores de riesgo: obesidad abdominal/ circunferencia de la cintura en hombre $>102 \mathrm{~cm}$ y en mujeres $>88 \mathrm{~cm}$; triglicéridos $\geq 150 \mathrm{mg} / \mathrm{dL}$; HDL colesterol $<40 \mathrm{mg} / \mathrm{dL}$ en varones y $<50 \mathrm{mg} / \mathrm{dL}$ en mujeres; presión arterial $\geq 130$ $185 \mathrm{mmHg}$, y glucosa en ayunas $\geq 110 \mathrm{mg} / \mathrm{dL}^{(5)}$.

La American Heart Association/National Heart Lung and Blood Institute (AHA/NHLBI) y Scientific Statement han adoptado los criterios de ATP III con la única variación de considerar glucosa $\geq 100 \mathrm{mg} / \mathrm{dL}$ y considerando a los triglicéridos y $\mathrm{HDL}$ en los pacientes que ingieren niacina o fibrato ${ }^{(6)}$. La definición de la International Diabetes Federation (IDF) publicada en 2005, considera como criterio imprescindible la obesidad central definida por valores de circunferencia abdominal específicos para cada etnia, en el caso de la población latinoamericana $\geq 90 \mathrm{~cm}$ para varones $\mathrm{y} \geq 80 \mathrm{~cm}$ para mujeres ${ }^{(7)}$.

Recientemente varias asociaciones, IDF, AHA, NHLBI, World Heart Foundation (WHF), International Association for the Study of Obesity (IASO) publicado en octubre de 2009 como Joint Interin Statement (JIS) han llegado a un consenso sobre los criterios diagnósticos de síndrome metabólico que emplean los criterios de ATPIII modificando: glicemia $\geq 100 \mathrm{mg} / \mathrm{dL}$ y el diámetro periumbilical de acuerdo a la raza. En este caso, para los sudamericanos establecen $\geq 90 \mathrm{~cm}$ en los varones $y$ $\geq 80 \mathrm{~cm}$ en las mujeres ${ }^{(7)}$.

En Perú, Seclén empleó los criterios de ATP III y encontró una prevalencia del SM de 14,9\%, Huamán en Trujillo encontró algo similar con una prevalencia de $16,3 \%$ sin diferencia de sexo; Pajuelo a nivel nacional encontró $16,8 \%$ con mayor frecuencia en mujeres $(26,4 \%)$ que en los varones $(7,2 \%) y$, por regiones, en Lima $20,7 \%$; resto de costa $21,5 \%$, y sierra rural $11,1 \%$. Soto, en Lambayeque, encontró una prevalencia según ATP III de $28,3 \%, 29,9 \%$ en mujeres y $23,1 \%$ en varones ${ }^{(8-11)}$.

La obesidad central es la medida del diámetro periumbilical o de la cintura; asimismo, ha sido identificado como un importante factor para desarrollo de diabetes tipo 2 y aterogénesis, por lo que es un elemento rutinario dentro de los distintos criterios diagnósticos del SM, sin embargo, no discrimina entre grasa intraabdominal (visceral) y subcutánea, esto, debido a que la grasa intraabdominal o visceral está relacionada con la intolerancia a la glucosa y a la hiperinsulinemia, como resultado de la resistencia a la insulina; por tal motivo es que se ha recurrido a unirla a la hipertrigliceridemia en la llamada "cintura hipertrigliceridémica" (CHTG) o "fenotipo de cintura hipertrigliceridémica" (12,13). La propuesta de la cintura hipertrigliceridémica está basada en el concepto de que la obesidad central y la dislipidemia son el núcleo patogénico del síndrome metabólico y, por lo tanto, son los componentes esenciales para identificar a aquellos sujetos con resistencia a la insulina y una gran tendencia aterogénica ${ }^{(14) .}$

Para el diagnóstico de CHTG, inicialmente, en Canadá, se utilizaron los siguientes criterios en varones: circunferencia de cintura $\geq 90 \mathrm{~cm}$ y nivel de triglicéridos $\geq$ $175 \mathrm{mg} / \mathrm{dL}$ (15). Posteriormente, para mujeres, se empleó: circunferencia de cintura $\geq 85 \mathrm{~cm}$ y nivel de triglicéridos $\geq$

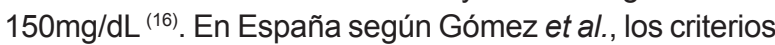
utilizados fueron: circunferencia de la cintura $\geq 94 \mathrm{~cm}$ para varones $y \geq 80 \mathrm{~cm}$ para mujeres $y$, nivel de triglicéridos $\geq 150 \mathrm{mg} / \mathrm{dL}$ para ambos sexos ${ }^{(17)}$. Según un estudio realizado por Lemiux en varones, la circunferencia de la cintura y triglicéridos en ayunas simultánea podrían ser utilizados como herramientas de detección de bajo costo para identificar a los hombres que presenten la tríada metabólica aterogénica (hiperinsulinemia, elevado apoliproteina $\mathrm{B}$, LDL pequeñas y densas), también es considerado por el estudio de Manrique Vera ya que relaciona la presencia de una medida antropométrica de fácil evaluación y el análisis auxiliar sérico de tan solo una fracción del perfil lipídico ${ }^{(15,18)}$.

En el presente estudio se consideraron dos criterios diagnóstico para SM, el de ATP III, el cual es ampliamente usado en múltiples estudios epidemiológicos, y el de JIS ya que armoniza los diferentes criterios de SM ${ }^{(19)}$. Asimismo se consideraron dos criterios diagnósticos para CHTG: el de Gómez, que fue realizado en población adulta española teniendo en cuenta sexo y edad, y cuyos criterios son similares a lo publicado en el 2005 por FID en el caso de población latinoamericana, diferente a otros estudios donde solo tomaron en cuenta población con sobrepeso y obesidad o en solo un sexo; y el de JIS, el cual se tomó en cuenta para efectos de comparación ${ }^{(17,19)}$.

El objetivo del estudio fue determinar la concordancia entre la cintura hipertrigliceridemia (CHTG) y el síndrome metabólico (SM) en adultos de Trujillo según criterios diagnósticos. 


\section{MATERIALES Y MÉTODOS}

\section{DISEÑO DEL ESTUDIO}

Se realizó un estudio observacional, prospectivo y de corte transversal. Se llevó a cabo en urbanizaciones de California, San Andrés, San Nicolás y Fátima del distrito de Trujillo, durante el periodo de mayo del año 2010 a mayo de 2012. Se incluyeron a 500 adultos (239 varones y 261 mujeres) entre 20 a 79 años, que aceptaron participar en el estudio. Se excluyó a gestantes, usuarios de fármacos hipolipemiantes o hiperlipemiantes, y aquellos que incumplieron o rechazaron participar.

\section{MUESTRA}

Para la selección de la muestra se utilizó el método aleatorio simple entre personas que acudieron y aceptaron participar en el estudio realizado en campañas de salud de despistaje de factores de riesgo metabólico en las urbanizaciones de California, San Andrés, San Nicolás y Fátima del distrito de Trujillo; posteriormente se obtuvo información para el estudio mediante una ficha de datos de cada participante. Un total de 2164 personas acudieron a las mencionadas campañas durante el periodo que duró la recolección de datos. De estos, solo 1364 personas aceptaron participar. El cálculo de tamaño de muestra inicialmente arrojó que era necesario un mínimo de 208 participantes, utilizándose como parámetros $Z \alpha=1,96$, prevalencia estimada de 0,20 , basándonos en un estudio previo ${ }^{(19)}$, y con una exactitud de 0,05 . Sin embargo, a efecto de una adecuada estratificación por edad y sexo, se amplió este número.

\section{DEFINICIÓN DE SÍNDROME METABÓLICO Y CHTG}

Luego de recolectar los datos se prosiguió con la aplicación de criterios para SM y CHTG de acuerdo a lo siguiente:

SM según ATPIII (SMATPIII). Presencia de al menos tres de los siguientes factores de riesgo: obesidad abdominal (circunferencia de la cintura en hombre $>102$ $\mathrm{cm}$ y en mujeres $>88 \mathrm{~cm}$ ); triglicéridos $\geq 150 \mathrm{mg} / \mathrm{dL}$; $\mathrm{HDL}$ colesterol $<40 \mathrm{mg} / \mathrm{dL}$ en varones $\mathrm{y}<50 \mathrm{mg} / \mathrm{dL}$ en mujeres; presión arterial $\geq 130 / 85 \mathrm{mmHg}$; glucosa en ayunas $\geq 110 \mathrm{mg} / \mathrm{dL}^{(19)}$.

SM según JIS(SM JIS). Presencia de, al menos, tres de los siguientes factores de riesgo: obesidad abdominal (circunferencia de la cintura en hombre $\geq 90 \mathrm{~cm}$ y en mujeres $\geq 80 \mathrm{~cm}$ ); triglicéridos $\geq 150 \mathrm{mg} / \mathrm{dL}$; HDL colesterol $<40 \mathrm{mg} / \mathrm{dL}$ en varones $\mathrm{y}<50 \mathrm{mg} / \mathrm{dL}$ en mujeres; presión arterial $\geq 130 / 85 \mathrm{mmHg}$; glucosa en ayunas $\geq$ $100 \mathrm{mg} / \mathrm{dL}^{(19)}$.
CHTG según Gómez. Circunferencia de la cintura $\geq$ $94 \mathrm{~cm}$ para varones $y \geq 80 \mathrm{~cm}$ para mujeres, y nivel de triglicéridos $\geq 150 \mathrm{mg} / \mathrm{dL}$ para ambos sexos ${ }^{(17)}$.

CHTG según JIS (CHTG JIS). Se empleó los criterios de JIS sobre obesidad central para efectos comparativos: cintura $\geq 90 \mathrm{~cm}$ para varones y el resto igual que Gómez.

\section{TÉCNICAS E INSTRUMENTOS DE RECOLECCIÓN DE DATOS}

A las personas que aceptaron participar en el estudio se les solicitó los siguientes datos: edad, sexo, antecedentes familiares diabetes, hipertensión y obesidad, consumo de tabaco y alcohol, fecha de inicio de último ciclo menstrual, síntomas asociados a diabetes e hipertensión (polifagia, polidipsia, poliuria, cefalea), antecedentes de diabetes e hipertensión; tipo, frecuencia, duración de actividad física y medicamentos utilizados cotidianamente; estos datos fueron completados por los autores en una ficha de datos. El peso y la talla fueron medidos por los autores con balanza y tallímetro debidamente calibrados, se pidió a los participantes que se quiten los zapatos, que vistan ropa ligera, que se pongan de pie con el distribuido igualmente en ambos pies, los brazos a los costados y con la mirada al frente. Asimismo, la circunferencia abdominal fue medida en un punto medio entre las crestas iliacas y el reborde costal inferior después de realizar una espiración completa ${ }^{(19)}$.

La presión arterial fue medida en posición sentada, con esfigmomanómetro de mercurio, usando el brazo no dominante. Después de $10 \mathrm{~min}$ de reposo, se tomaron tres lecturas y se utilizó el promedio de las dos últimas. La presión sistólica fue registrada coincidiendo con la fase I de Korotkoff y la diastólica con la fase $\mathrm{V}$ de Korotkoff ${ }^{(20)}$.

La toma de muestras sanguíneas fue en condiciones de ayunas de 10 a $12 \mathrm{~h}$. Se les extrajo una muestra de sangre venosa del antebrazo en posición sentada, en recipiente sin anticoagulante, se separó el suero por centrifugación a 3500 rpm durante 5 min. Se determinó la glicemia, el colesterol y triglicéridos por métodos enzimáticos empleando reactivos estandarizados, el HDL colesterol por precipitación con sulfato de Dextran y el LDL se calculó usando la fórmula de Friedwald (21-23).

\section{ANÁLISIS DE DATOS}

Se estimó la frecuencia de la CHTG según criterios de Gómez y JIS en general, por sexo y por grupos etarios; se compararon las proporciones empleando el test $Z$ y la comparación de medias con la prueba $T$ de Student. Se consideró significativo un $p<0,05$. La concordancia entre la frecuencia de CHTG y SM se hizo aplicando 
el coeficiente kappa. Se empleó el programa EPIDAT v 3.1 desarrollado por la Organización Panamericana de la Salud. Para la interpretación del coeficiente kappa se consideró los siguientes intervalos: $<0,20$ : pobre; 0,21 0,40: débil; 0,41-0,60: moderada; 0,61-0,80: buena, y $0,81-1,00$ : muy buena ${ }^{(24)}$.

\section{ASPECTOS ÉTICOS}

El estudio fue aprobado por el Comité de Investigación de la Facultad de Medicina de la Universidad Nacional de Trujillo. El estudio se realizó en el marco de las recomendaciones de Helsinski para la investigación biomédica, así como el Código de Ética del Colegio Médico del Perú. Los participantes firmaron el acta de consentimiento informado, previa información de los objetivos y propósitos del estudio. La encuesta fue anónima.

\section{RESULTADOS}

Se utilizaron datos de 500 personas residentes en la ciudad de Trujillo, de las cuales 239 fueron varones y 261 mujeres, según criterios propuestos. En la Tabla 1 se muestran las características generales de las personas en el estudio. No hubo diferencia en edad, entre ambos sexos. Los varones tuvieron significativamente mayor IMC, cintura, presión arterial sistólica, presión arterial diastólica, concentración de triglicéridos y glucosa. Las mujeres, en cambio, tuvieron mayor concentración de colesterol, LDL y HDL. Es propicio mencionar que la frecuencia del SM ATP III en varones fue de $21,8 \%$ y en mujeres fue $22,2 \%$. La frecuencia del SM JIS en varones fue $37,2 \%$ y en mujeres fue de $39,8 \%$.En cuanto a la población total se encontró que la frecuencia del SM ATPIII fue de $22 \%$ y del SM JIS fue $38,6 \%$.

En la Tabla 2 se muestra la frecuencia del fenotipo CHTG según Gómez, donde se observa que fue de $30,0 \%$ en el total de la población, en varones 29,7 \% y

Tabla 1. Características generales de la población estudiada

\begin{tabular}{lccc}
\hline \multicolumn{1}{c}{ Criterios } & Varones & Mujeres & $\boldsymbol{p}$ \\
\hline Edad (años) \pm D.E. & $48,92 \pm 17,74$ & $49,92 \pm 16,83$ & 0,253 \\
IMC $\left(\mathrm{kg} / \mathrm{m}^{2}\right)$ & $26,78 \pm 3,90$ & $25,46 \pm 4,04$ & $<0,001$ \\
Cintura $(\mathrm{cm})$ & $93,72 \pm 10,34$ & $83,46 \pm 9,98$ & $<0,001$ \\
PAS $(\mathrm{mmHg})$ & $120,62 \pm 14,54$ & $117,45 \pm 17,45$ & 0,014 \\
PAD (mmHg) & $75,86 \pm 9,42$ & $73,74 \pm 10,18$ & 0,008 \\
Triglicéridos (mg/dL) & $177,36 \pm 109,50$ & $147,10 \pm 87,80$ & $<0,001$ \\
HDL (mg/dL) & $46,23 \pm 7,77$ & $49,79 \pm 8,16$ & $<0,001$ \\
Glucosa (mg/dL) & $101,52 \pm 38,40$ & $96,19 \pm 30,97$ & 0,040 \\
Colesterol (mg/dL) & $211,18 \pm 40,85$ & $223,10 \pm 47,35$ & 0,001 \\
LDL (mg/dL) & $129,54 \pm 37,33$ & $143,46 \pm 43,53$ & $<0,001$ \\
\hline
\end{tabular}

D.E.: desviación estándar
Tabla 2. Frecuencia de cintura hipertrigliceridémica según criterios en la población adulta de Trujillo, por edad y sexo

\begin{tabular}{|c|c|c|c|c|c|c|}
\hline & \multirow{2}{*}{ Total } & \multicolumn{2}{|c|}{$\begin{array}{l}\text { Según } \\
\text { Gómez }\end{array}$} & \multicolumn{2}{|c|}{$\begin{array}{c}\text { Según } \\
\text { JIS }\end{array}$} & \multirow[t]{2}{*}{$p$} \\
\hline & & $\mathbf{N}$ & $(\%)$ & $N$ & $(\%)$ & \\
\hline \multicolumn{7}{|l|}{ Varones } \\
\hline $20-39$ & 75 & 19 & $(25,3)$ & 24 & $(32,0)$ & 0,470 \\
\hline $40-59$ & 85 & 26 & $(30,6)$ & 35 & $(41,2)$ & 0,201 \\
\hline $60-79$ & 79 & 26 & $(32,9)$ & 32 & $(40,5)$ & 0,409 \\
\hline Total & 239 & 71 & $(29,7)$ & 91 & $(38,1)$ & 0,066 \\
\hline \multicolumn{7}{|l|}{ Mujeres } \\
\hline $20-39$ & 86 & 12 & $(14,0)$ & 12 & $(14,0)$ & * \\
\hline $40-59$ & 88 & 39 & $(44,3)$ & 39 & $(44,3)$ & * \\
\hline $60-79$ & 87 & 28 & $(32,2)$ & 28 & $(32,2)$ & * \\
\hline Total & 261 & 79 & $(30,3)$ & 79 & $(30,3)$ & * \\
\hline \multicolumn{7}{|c|}{ Población } \\
\hline $20-39$ & 161 & 31 & $(19,3)$ & 36 & $(22,4)$ & 0,583 \\
\hline $40-59$ & 173 & 65 & $(37,6)$ & 74 & $(42,8)$ & ${ }^{\prime} 0,380$ \\
\hline $60-79$ & 166 & 54 & $(32,5)$ & 60 & $(36,1)$ & 0,563 \\
\hline Total & 500 & 150 & $(30,0)$ & 170 & $(34,0)$ & 0,198 \\
\hline
\end{tabular}

*No se muestra el valor $p$ en mujeres, ya que en ambas definiciones de cintura hipertrigliceridémica presentan los mismos valores

en mujeres $30,3 \%$, sin diferencia significativa. La edad afectó significativamente la frecuencia en varones y en mujeres siendo más frecuente en varones entre 60-79 años con 32,9 \% y en mujeres entre 40-59 años con $44,3 \%$. En la misma tabla se muestra la frecuencia del fenotipo CHTG JIS que fue de $34,0 \%$ en el total de la población, en varones $38,1 \%$ y en mujeres $30,3 \% \sin$ diferencia significativa. La edad afectó significativamente la frecuencia en mujeres y en el grupo de 20-39 años fue significativamente mayor en varones.

En la Tabla 3 se muestra la concordancia entre las distintas definiciones empleadas de CHTG y SM donde se utilizó el test $\mathrm{k}$. La concordancia entre el fenotipo CTHG según Gómez y el SMATP III en varones, en mujeres y en la población total fue moderada; siendo buena en varones de 60 a 79 años con $\mathrm{K}=0,62$. La concordancia entre el fenotipo CTHG según Gómez y el SM JIS fue buena en varones, mujeres y en el total de la población; teniendo buena concordancia entre 20 a 39 años en población total con $\mathrm{K}=0,74$. La concordancia entre CTHG JIS y el SMATP III en varones, mujeres y población total fue moderada; siendo buena entre varones de 60 a 79 años con $\mathrm{K}=070$ y en población total con el mismo rango de edades con $K=063$. La concordancia entre CTHG y SM según JIS fue buena en varones, mujeres y en el total de la población; solo hay moderada concordancia en mujeres de 60 a 79 años con $\mathrm{K}=050$ y población total del mismo rango de edad con $\mathrm{K}=058$. En las mujeres el test $k$ es el mismo entre CHTG según Gómez frente a SMATP III, y CHTG JIS frente a SMATP III con $\mathrm{K}=0,60$; 
Tabla 3. Concordancia entre la frecuencia de cintura hipertrigliceridémica y síndrome metabólico, Trujillo, Perú.

\begin{tabular}{|c|c|c|c|c|c|c|c|c|}
\hline & \multicolumn{2}{|c|}{$\begin{array}{c}\text { C-HTG Gómez frente a } \\
\text { SM ATP III }\end{array}$} & \multicolumn{2}{|c|}{$\begin{array}{c}\text { C-HTG Gómez frente a } \\
\text { SM JIS }\end{array}$} & \multicolumn{2}{|c|}{$\begin{array}{c}\text { C-HTG JIS frente a } \\
\text { SM ATP3 }\end{array}$} & \multicolumn{2}{|c|}{$\begin{array}{c}\text { CTHG JISS frente a } \\
\text { SM JISS }\end{array}$} \\
\hline & kappa & (IC 95\%) & kappa & (IC 95\%) & kappa & (IC 95\%) & kappa & (IC 95\%) \\
\hline \multicolumn{9}{|l|}{ Varones } \\
\hline $20-39$ & 0,43 & $(0,19-0,67)$ & 0,75 & $(0,57-0,92)$ & 0,4 & $(0,20-0,61)$ & 0,74 & $(0,57-0,90)$ \\
\hline $40-59$ & 0,55 & $(0,35-0,75)$ & 0,53 & $(0,35-0,71)$ & 0,43 & $(0,24-0,61)$ & 0,61 & $(0,44-0,78)$ \\
\hline $60-79$ & 0,62 & $(0,43-0,81)$ & 0,57 & $(0,39-0,76)$ & 0,7 & $(0,54-0,86)$ & 0,69 & $(0,52-0,85)$ \\
\hline Total & 0,55 & $(0,43-0,67)$ & 0,61 & $(0,50-0,71)$ & 0,53 & $(0,42-0,63)$ & 0,68 & $(0,58-0,77)$ \\
\hline \multicolumn{9}{|l|}{ Mujeres } \\
\hline $20-39$ & 0,51 & $(0,22-0,80)$ & 0,71 & $(0,49-0,91)$ & 0,51 & $(0,22-0,80)$ & 0,71 & $(0,49-0,93)$ \\
\hline $40-59$ & 0,6 & $(0,43-0,76)$ & 0,66 & $(0,51-0,81)$ & 0,6 & $(0,43-0,76)$ & 0,66 & $(0,51-0,81)$ \\
\hline $60-79$ & 0,56 & $(0,37-0,75)$ & 0,5 & $(0,33-0,67)$ & 0,56 & $(0,37-0,75)$ & 0,50 & $(0,33-0,67)$ \\
\hline Total & 0,6 & $(0,49-0,71)$ & 0,64 & $(0,55-0,74)$ & 0,6 & $(0,49-0,71)$ & 0,64 & $(0,55-0,74)$ \\
\hline \multicolumn{9}{|c|}{ Población } \\
\hline $20-39$ & 0,47 & $(0,29-0,65)$ & 0,74 & $(0,60-0,87)$ & 0,45 & $(0,28-0,62)$ & 0,73 & $(0,60-0,86)$ \\
\hline $40-59$ & 0,58 & $(0,46-0,71)$ & 0,61 & $(0,49-0,72)$ & 0,52 & $(0,39-0,64)$ & 0,64 & $(0,53-0,75)$ \\
\hline $60-79$ & 0,59 & $(0,46-0,72)$ & 0,53 & $(0,48-0,66)$ & 0,63 & $(0,51-0,76)$ & 0,58 & $(0,46-0,70)$ \\
\hline Total & 0,58 & $(0,50-0,66)$ & 0,63 & $(0,55-0,70)$ & 0,56 & $(0,48-0,64)$ & 0,66 & $(0,59-0,73)$ \\
\hline
\end{tabular}

C-HTG: cintura hipertrgliceridémica, SM: cindrome metabólico, JIS: Joint Interin Statement

asimismo el test k es el mismo entre CHTG según Gómez frente a SM JIS, y CHTG JIS frente a SM JIS, con $k=0,64$.

\section{DISCUSIÓN}

Las características de la población estudiada y la diferencia en los sexos son semejantes a lo descrito anteriormente, salvo la glucosa que aparece ligeramente mayor en los varones ${ }^{(19)}$. En el presente estudio, la frecuencia de CHTG según Gómez, en el total de la población estudiada, fue de $30 \%$ en 500 individuos de Trujillo de 20 a 79 años, mayor a lo reportado por el mismo Gómez que fue de $14,5 \%$ en 2270 individuos de España de 18 a 80 años; así mismo, la frecuencia en varones fue de $29,71 \%$ y en mujeres de $30,3 \%$; a diferencia de lo encontrado por Gómez que fue mayor en varones $(18,2 \%)$ que en mujeres $(10,8 \%)$; además, se encontró el incremento con la edad, siendo mayor en varones de 60 a 79 años y en mujeres de 40 a 59 años, muy semejante a lo reportado por Gómez ${ }^{(8,17)}$. La mayor frecuencia en varones y el incremento con la edad ha sido reportada por otros autores ${ }^{(25,26)}$.

En Perú, en un estudio retrospectivo de casos y controles, realizado por Manrique en una población de 426 pacientes adultos con sobrepeso y obesidad de 18-79 años, se encontró que la frecuencia de CHTG se presentó en $41,8 \%$, y cuando fue modificado en cuanto al punto de corte de triglicéridos (triglicéridos $\geq 150 \mathrm{mg} / \mathrm{dL}$ ) fue $56,3 \%$; este último resultado fue mayor a lo encontrado por este estudio, esto se puede explicar pues en el estudio hecho por Manrique tuvo como población a personas con sobrepeso y obesidad ${ }^{(18)}$.

La frecuencia de CHTG JIS en el total de la población estudiada fue de $34 \%$ en 500 individuos de Trujillo de 20 a 79 años; así mismo, la frecuencia es mayor en varones debido a que el requisito para la cintura es menor en donde se considera $\geq 90 \mathrm{~cm}$.

Gómez encuentra que la concordancia entre CHTG según Gómez y SMATP III fue moderada $(k: 0,51)$ semejante al actual estudio (k: 0,58); sin embargo, la concordancia entre CHTG frente a SM según JIS es buena $(k: 0,66)$ lo cual es explicable porque ambos tienen el mismo criterio para la cintura así como CHTG según Gómez frente a SM JIS con k=0,64 a pesar que difieren en el criterio de cintura para varones ${ }^{(17) .}$

En un estudio realizado en Malaysia, donde se tuvo como criterio de CHTG a la circunferencia de la cintura en hombre $>90 \mathrm{~cm}$ y en mujeres $>80 \mathrm{~cm}$, así como el nivel de triglicéridos $>150 \mathrm{mg} / \mathrm{dL}$; se encontró una buena concordancia entre SM ATP III y CHTG, este resultado difiere al presente estudio donde se encuentra moderada concordancia ya que los criterios utilizados son diferentes ${ }^{(27)}$.

En una revisión realizado por Scarsella en el 2003, se propone que la CHTG puede identificar a un sector significativo de portadores de la triada metabólica aterogénica y presentar enfermedad coronaria, asimismo, se menciona que con la edad se produce 
una acumulación de tejido intraabdominal, esto tiene similitud con el presente estudio donde la frecuencia de la CTHG, según Gómez y JIS, aumenta con la edad en varones no siendo lo mismo en mujeres ${ }^{(12)}$.

La limitación en el estudio fue la captación de participantes a través de campañas y no mediante un muestreo por etapas como en otros estudios. Se concluye que la concordancia fue buena entre la CHTG con ambos criterios y SM según JIS.
Contribuciones de autoría: ELR, KCM, MRM y JHS han participado en la concepción y diseño del trabajo. ELR, KCM y JHS participó en la revisión y redacción parcial del artículo. KCM, MRM y JHS realizaron la recolección, análisis de datos y asesoría estadística. Todos los autores aprobaron la versión final a publicar.

Fuentes de financiamiento: autofinanciado.

Conflictos de interés: los autores declaran no tener conflictos de interés.

\section{REFERENCIAS BIBLIOGRÁFICAS}

1. Grundy SM. Metabolic syndrome pandemic. Arterioscler Thromb Vasc Biol. 2008 Apr;28(4):629-36. doi: 10.1161/ ATVBAHA.107.151092.

2. Reaven GM. Role of insulin resistance in human disease. Diabetes. $1989 ; 37(12): 1595-1607 . \quad$ doi: 10.2337/diab.37.12.1595.

3. Isomaa $\mathrm{B}$, Almgren P, Tuomi T, Forsén B, Lahti K, Nissén M, et al. Cardiovascular morbidity and mortality associated with the metabolic syndrome. Diabetes Care. 2001 Apr;24(4):683-9.

4. Haffner SM, Valdez RA, Hazuda HP, Mitchell BD, Morales PA, Stern MP. Prospective analysis of the insulin-resistance syndrome (syndrome X). Diabetes. 1992 Jun; $41(6): 715-22$.

5. Expert Panel on Detection, Evaluation, and Treatment of High Blood Cholesterol in Adults. Executive Summary of The Third Report of The $\mathrm{Na}$ tional Cholesterol Education Program (NCEP) Expert Panel on Detection, Evaluation, And Treatment of High Blood Cholesterol In Adults (Adult Treatment Panel III). JAMA. 2001 May 16;285(19):2486-97.

6. Grundy SM, Cleeman JI, Daniels SR, Donato KA, Eckel RH, Franklin BA, et al. Diagnosis and management of the metabolic syndrome: an American Heart Association/National Heart, Lung, and Blood Institute scientific statement: Executive Summary. Circulation 2005;112:e285-e290

7. Alberti KG, Eckel RH, Grundy SM, Zimmet PZ, Cleeman JI, Donato KA, et al. Harmonizing the metabolic syndrome: a joint interim statement of the International Diabetes Federation Task Force on Epidemiology and Pre- vention; National Heart, Lung, and Blood Institute; American Heart Association; World Heart Federation; International Atherosclerosis Society; and International Association for the Study of Obesity. Circulation. 2009 Oct 20;120(16):1640-5. doi: 10.1161/ CIRCULATIONAHA.109.192644.

8. Seclén S, Villena A, Larrad MT, Gamarra D, Herrera B, Pérez CF, et al. Prevalence of the metabolic syndrome in the mestizo population of Peru. Metab Syndr Relat Disord. 2006 Spring;4(1):1-6. doi: 10.1089/ met.2006.4.1.

9. Huamán SJ. Prevalencia del Síndrome Metabólico en población adulta de Trujillo, según edad, género y algunos factores asociados. Acta Med Orreg Hampi Runa. 2007;7(1):4-10.

10. Pajuelo J, Sánchez J. El síndrome metabólico en adultos, en el Perú. An Fac Med Lima. 2007;68(1):38-46.

11. Soto V, Vergara E, Neciosusp E. Prevalencia y factores de riesgo de síndrome metabólico en población adulta del departamento de Lambayeque, Perú 2004. Rev Per Med Exp Salud Publica. 2005;22(4):254-61.

12. Scarsella C, Després JP. Tratamiento de la obesidad: necesita de centrar la atención en los pacientes de alto riesgo caracterizados por la obesidad abdominal. Cad Saude Publica. 2003;19 Suppl 1:S7-19.

13. Després JP, Lemieux I, Bergeron J, Pibarot P, Mathieu P, Larose E, et al. Abdominal obesity and the metabolic syndrome: contribution to global cardiometabolis risk. Arterioscler Thromb Vasc Biol.2008 Jun;28(6):1039-49. doi: 10.1161/ATVBAHA.107.159228.
14. Carr DB, Utzschneider KM, Hull RL, Kodama K, Retzlaff BM, Brunzell JD, et al. Intra-abdominal fat is a major determinant of the National Cholesterol Education Program Adult Treatment Panel III criteria for the metabolic syndrome. Diabetes. 2004 Aug;53(8):2087-94.

15. Lemieux I, Pascot A, Couillard C, Lamarche B, Tchernof A, Alméras N, et al. Hypertriglyceridemic waist: a marker of the atherogenic metabolic triad (hyperinsulinemia; hyperapolipoprotein B; small, dense LDL) in men? Circulation. 2000 Jul 11;102(2):179-84.

16. Arsenault BJ, Lemieux I, Després JP, Wareham NJ, Kastelein JJ, Khaw KT, et al. The hypertriglyceridemic-waist phenotype and the risk of coronary artery disease: results from the EPICNorfolk prospective population study. CMAJ. 2010 Sep 21;182(13):1427-32. doi: 10.1503/cmaj.091276.

17. Gomez-Huelgas R, Bernal-López MR, Villalobos A, Mancera-Romero J, Baca-Osorio AJ, Jansen S, et al. Hypertriglyceridemic waist: an alternative to the metabolic syndrome? Results of the IMAP Study (multidisciplinary intervention in primary care). Int $\mathrm{J}$ Obes (Lond). 2011 Feb;35(2):292-9. doi: 10.1038/ijo.2010.127.

18. Manrique-Vera A, Manrique-Hurtado $\mathrm{H}$. Frecuencia del fenotipo 'cintura hipertrigliceridémica' y su asociación con el síndrome con el síndrome metabólico en adultos con sobrepeso y obesidad. Rev Soc Peru Med Interna. 2010;23(3):92-7.

19. Galarreta Aima CI, Donet Mostacero JA, Huamán Saavedra JJ. Síndrome metabólico en la población adulta 
de Trujillo de acuerdo a diferentes definiciones. Acta Med Peruana. 2009;26(4):217-25.

20. Chobanian AV, Bakris GL, Black HR, Cushman WC, Green LA, Izzo JL et al. The Seventh Report of the Joint National Committee on Prevention, Detection, Evaluation and Treatment of High Blood Pressure: the JNC 7 report. JAMA. 2003 May 21;289(19):2560-72.

21. Wiener Lab. Vademecum para Laboratorio Clínico. Rosario: Wiener Lab; 2002.

22. Warnick GR, Benderson J, Albers JJ. Dextran sulfate-Mg2+ precipitation procedure for quantitation of highdensity-lipoprotein cholesterol. Clin Chem. 1982 Jun;28(6):1379-88.
23. Friedwald WT, Levy RI, Fredickson DS. Estimation of the concentration of low density lipoprotein colesterol in plasma without use of preparation ultracentrifuge. Clin Chem. 1972 Jun;18(6):499-502.

24. López de Ullibarri Galparsoro I, Pita Fernández S. Medidas de concordancia: el índice Kappa. Cad Aten Primaria. 1999;6:169-71.

25. Kahn HS, Valdez R. Metabolic risks identified by the combination of enlarged waist and elevated triacylglycerol concentration. Am J Clin Nutr. 2003 Nov;78(5):928-34.

26. Solati M, Ghanbarian A, Rahmani M, Sarbazi N, Allahverdian S, Azizi F. Cardiovascular risk factors in males with hypertriglycemic waist (Tehran Lipid and Glucose Study). Int J Obes Relat Metab Disord. 2004 May;28(5):706-9.

27. Zainuddin LR, Isa N, Muda WM, Mohamed HJ. The prevalence of metabolic syndrome according to various definitions and hypertriglyceridemic-waist in Malaysian adults. Int J Prev Med. 2011 Oct;2(4):229-37.

Correspondencia: Edgardo Linares Reyes Dirección: Fermin Tangüis N. ${ }^{\circ} 380$, Trujillo, Perú

Correo electrónico:dedalo83_8@hotmail.com

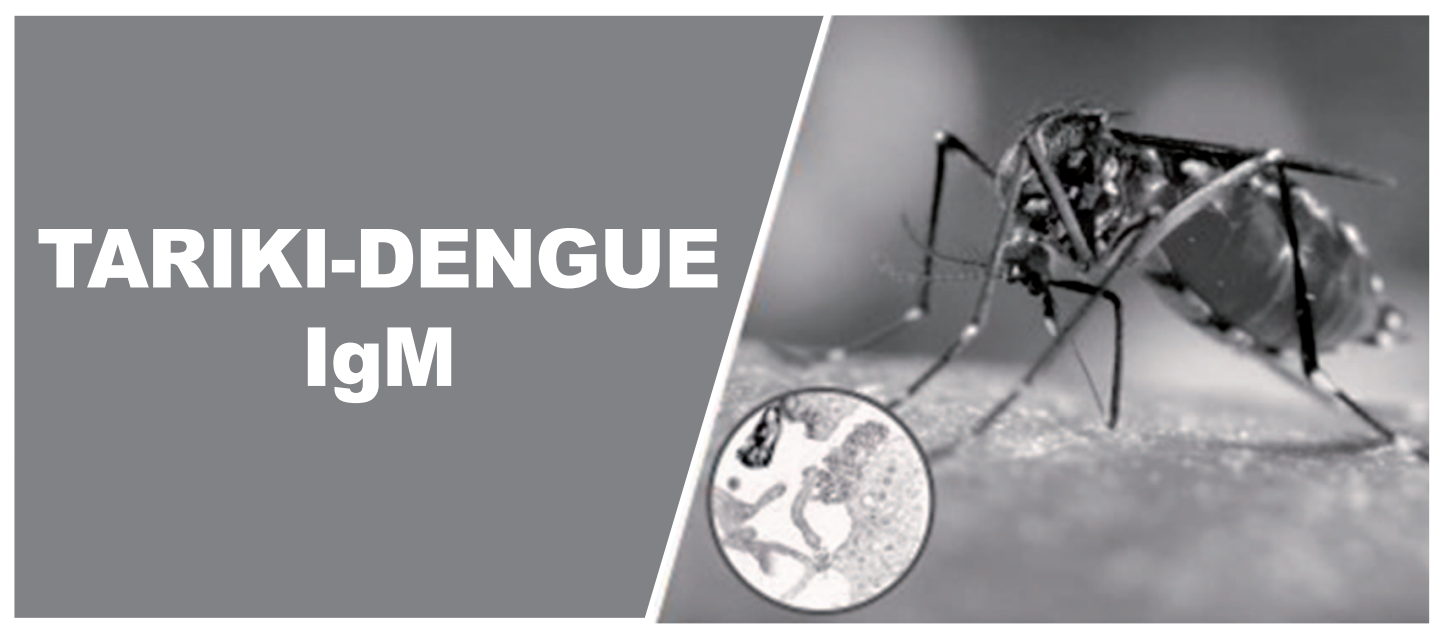

\section{ELISA de CAPTURA IgM DENGUE}

\section{TARIKI: disponible en el Perú}

Kit para la determinación de anticuerpos IgM anti Dengue, desarrollado y producido por el Instituto Nacional de Salud, Perú INS/MINSA 